\title{
Research on AM CVn stars: ASASSN-14ei and CR Boo
}

\author{
Keisuke Isogai ${ }^{* \dagger}$ \\ University of Kyoto, Japan \\ E-mail: isogaidkusastro.kvoto-u.ac.ip
}

\section{Taichi Kato}

University of Kyoto, Japan

E-mail: Ekatodkusastro,kyoto-u.ac. 70

\section{Daisaku Nogami}

University of Kyoto, Japan

E-mail: hogamiekusastro.kyoto-u.ac.jp

\section{Tomohito Ohshima}

Nishi-Harima Astronomical Observatory, University of Hyogo, Japan

E-mail: bhshimadnhao.ip

\section{Akira Imada}

Kwasan-Hida Observatory, University of Kyoto, Japan

E-mail: a_imadadkwasan.kyoto-u.ac.jp

\begin{abstract}
We report on time-resolved photometries of 2014-2015 superoutbursts of AM CVn-type objects, ASASSN-14ei and CR Boo. ASASSN-14ei is the first object which showed WZ Sge-like multiple rebrightening phase with a super long duration (at least 6 months). The superhump period had gradually decreased throughout the rebrightening phase. We suppose that the decreasing period reflects a shrinking disk. During the 2014 April superoutburst of CR Boo, we detected growing superhumps (stage A superhumps) whose period was $0.017669(24) \mathrm{d}$. By using the superhump period, the mass ratio was estimated to be $0.101(4)$, which is consistent with that expected by the theoretical evolutionary model.
\end{abstract}

The Golden Age of Cataclysmic Variables and Related Objects - III

7-12 September 2015

Palermo, Italy

* Speaker.

$\dagger$ Observers: Pavol A. Dubovsky, Kiyoshi Kasai, Lew Cook, Seiichiro Kiyota, Yutaka Maeda, Shawn Dvorak, Josch Hambsch, Hiroshi Itoh, Arto Oksanen, Gianluca Masi, Novak, Osaka Kyoiku U. team, Crimean Astrophysics Observatory Team, Berto Monard, Kyoto U. team, AAVSO collabolations. 


\section{Introduction}

AM CVn-type objects are a subclass of cataclysmic variables (CVs) that composed of a white dwarf primary and a mass transferring secondary. They are characterized by absence of hydrogen lines in spectra and their ultra-short orbital periods of $\sim 5-65 \mathrm{~min}$. Because of these features, AM CVn-type objects are expected as one of the most promising source of gravitational wave radiation and the progenitor of some of the type Ia supernovae. Hence, it is important to inspect the evolutionary model of AM CVn-type objects (For a review, see [四]). It is known that AM CVn-type objects whose orbital periods are $\sim 1300-2500 \mathrm{sec}$ show superoutbursts and superhumps. By using the method developed by [], we can estimate the mass ratio $q$ if the stage A superhump period and the orbital period $P_{\text {orb }}$ are well determined (for definition of stage A superhump, see [ [3]).

It is known that a part of AM CVn-type objects shows "rebrightenings" like WZ Sge-type objects (e.g. [团]), which is a subclass of hydrogen-rich CVs (for a review of WZ Sge-type, see [回]). Here, we regarded outbursts which seem to be triggered by a superoutburst as rebrightenings, also called "echo outbursts". There are basically two interpretations of the mechanism of rebrightenings. It is assumed that rebrightenings are caused by (1) the disk instability model: mass flow from the outer remnant disk and temporarily increased viscosity of the disk, which may involve with

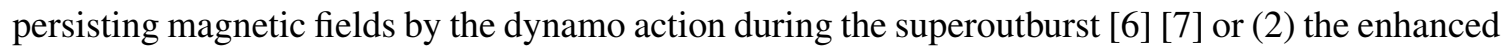
mass transfer model: variations of the mass transfer rate from the secondary by the irradiation i.e. VY Scl-type variations [ [8] [Q].

We report on the 2014-2015 superoutburst of a newly confirmed AM CVn-type object, ASASSN14ei in section [3]. This system showed repetitive rebrightening phase with the super long duration of 6 months. We also report on the 2014 April superoutburst of CR Boo in section 因. Note that we had already published the paper about the 2014 and 2015 superoutbursts of CR Boo with [एत]], thus we summarize the result of the 2014 superoutburst here.

\section{Observations and Analysis}

Our time-resolved photometric campaigns were carried out in 2014-2015 for ASASSN-14ei and in 2014 April-May for CR Boo by the VSNET Collaborations [ㅁ]]. The data were mainly acquired with $30 \mathrm{~cm}$-class telescopes. We also used the public data of American Association of Variable Star Observers (AAVSO ${ }^{1}$ ), All-Sky Automated Survey for Supernovae (ASASSN[ए2]) and Catalina Real-Time Transient Survey (CRTS[[3]]) for ASASSN-14ei. The time of the observations were corrected to Barycentric Julian Date (BJD), and we applied zero-point corrections to each observer by adding constants. For analyzing the superhump periods, we used the phase dispersion minimization (PDM) method [14]]. Before the analyses, we subtracted the global trend of the light curve by using smoothed light curve obtained by locally weighted polynomial regression (LOWESS [[15]). We also drawn the $O-C$ diagrams to detect the stage transitions of superhumps.

\section{ASASSN-14ei}

ASASSN-14ei was discovered by ASAS-SN at $V \sim 15.8 \mathrm{mag}$ on 2014 July $21.36 \mathrm{UT}$, and the

\footnotetext{
${ }^{1}$ http://www.aavso.org/data/download/
} 
previous outburst was reported at $V \sim 11.9$ mag on $10.38 \mathrm{UT}[\mathbb{1 6}]$. According to CRTS data, there is no past outburst and the mean magnitude in quiescence is $C V \sim 18.05$ mag. Optical spectroscopic observations were carried out on July 28.4 UT and August 01.4 UT, then He I and He II emission lines were confirmed[ㅁ] ].
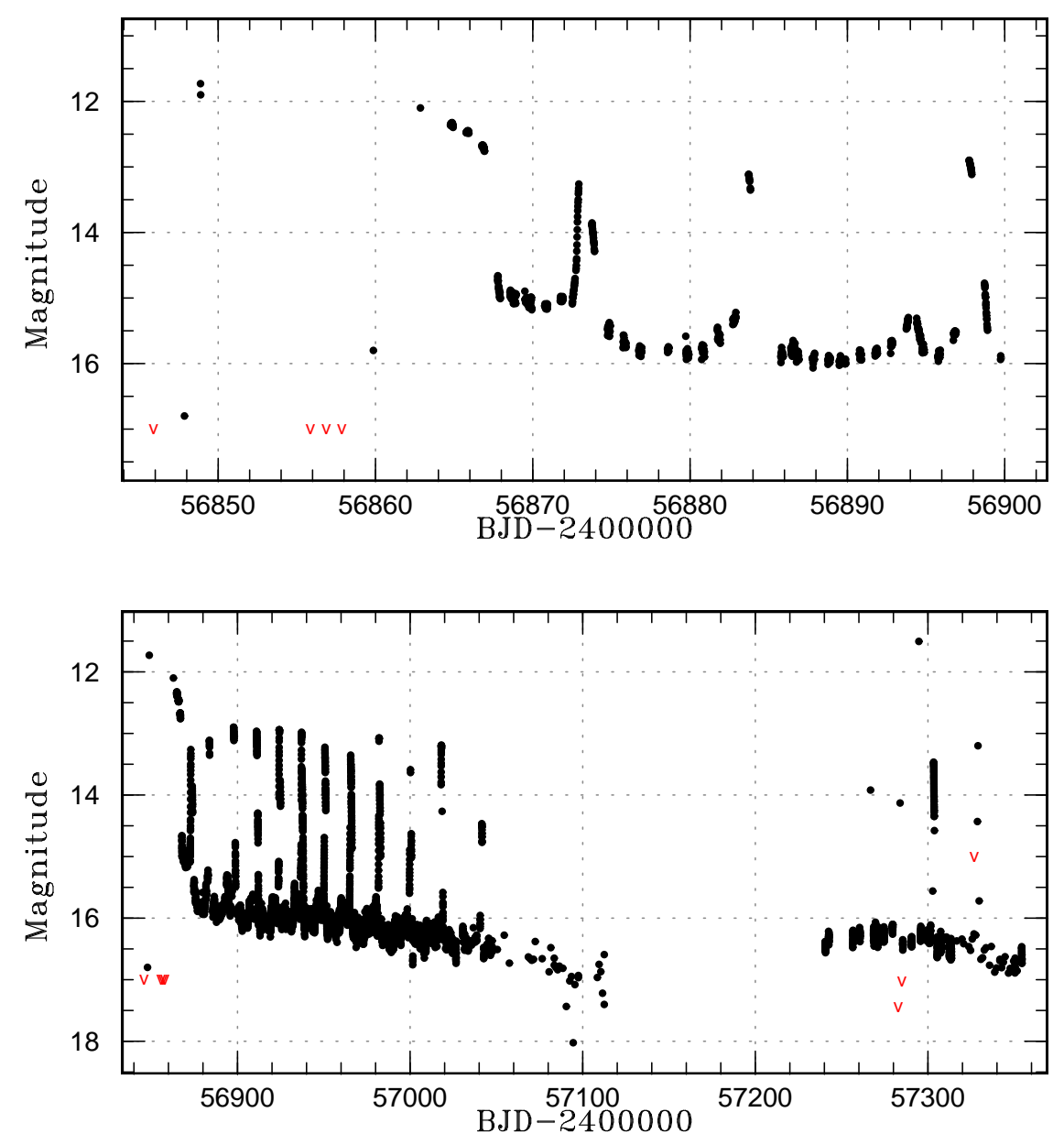

Figure 1: The initial part of the light curve (Upper) and the overall one (Lower) of the 2014-2015 superoutburst of ASASSN-14ei. V-shaped red points represent the upper limits by ASAS-SN and visual observations.

\subsection{Overall Light Curve}

Upper panel of figure $\amalg$ shows the initial part of the light curve (BJD 2456847.86-2456900.00). V-shaped red points indicate the upper limit by ASAS-SN and visual observations. Between the first outburst (BJD 2456849) and the second one (BJD 2456860-2456877), we can see that the object temporarily faded to $V>17.0$ mag. Because the second outburst showed the plateau phase, we regarded the second outburst as a main superoutburst, and regarded the third and the following ones as rebrightenings. It is unclear whether the first outburst is a precursor one or a part of the main superoutburst. If the latter is the case, this outburst represents a double (type-E) superoutburst like some of WZ Sge-type objects (for more detail, see e.g. [1[8]). 


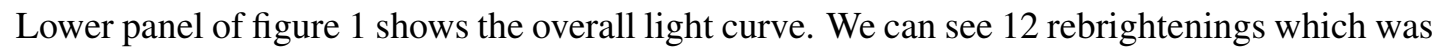
superimposed on a very long fading tail in 6 months after the main superoutburst. The duration of each rebrightening was about 1-2 days. Although the object also showed 5 outbursts after the seasonal gap (BJD 2457240-2457354), we did not regard these outbursts as rebrightenings because the behavior in the gap (BJD 2457113-2457240) is unclear. Note that the mean magnitude in the quiescence before the first outburst was $\sim 18.05$ mag, whereas the fading tail after the main superoutburst has been always brighter except a dip on BJD 2457095.

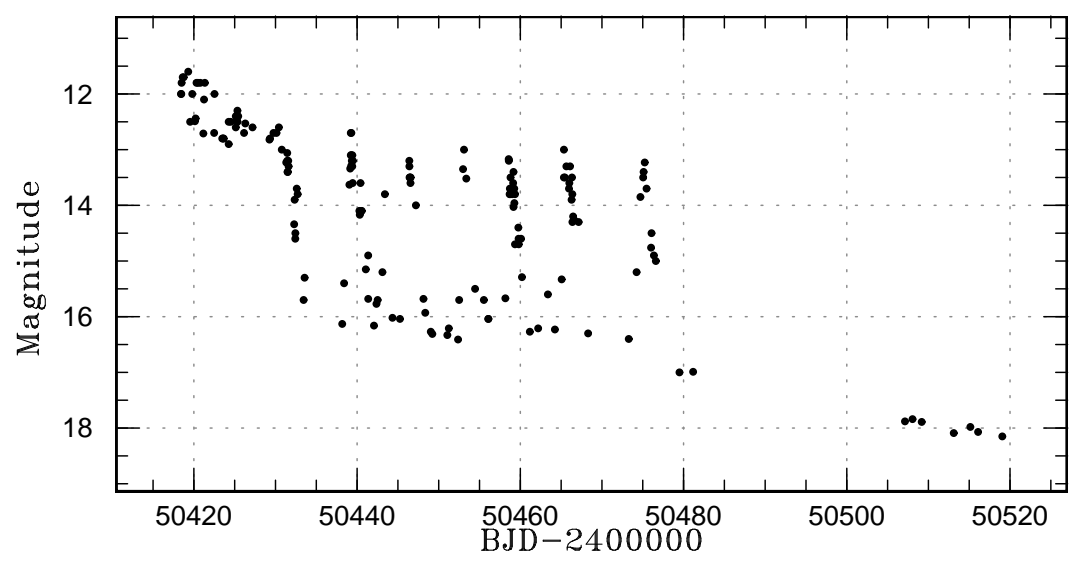

Figure 2: Light curve of the 1996 superoutburst of EG Cnc[प्प]. After the main superoutburst, the object showed a fading tail with 6 rebrightenings.

\subsection{WZ Sge-like Rebrightenings}

The 1996 superoutburst of the WZ Sge-type object EG Cnc also showed multiple rebrighten-

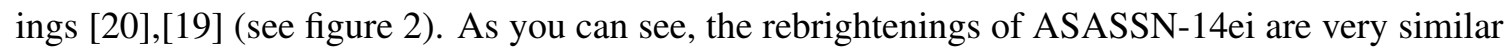
to ones of EG Cnc except the time scale. The recurrence times of rebrightenings of ASASSN-14ei and EG Cnc were about 15 and 7 days, respectively.Furthermore, the duration of rebrightening phases of ASASSN-14ei and EG Cnc were about 174 and 42 days, respectively. It is the first case that such extreme long duration of rebrightening phase was discovered including hydrogen-rich CVs.

\subsection{Superhumps and Rebrightening Model}

The light curve had shown double-peak modulations throughout our observations. We divided the fading tail into 3 sections and analyzed the period by PDM method in order to inspect the period variation of superhumps. The results are indicated in figure 13 and $\mathbf{A}$. The estimated mean periods are 0.0297948(3) $\mathrm{d}$ in the first section (BJD 2456868-2456928), 0.0297746(3) $\mathrm{d}$ in the second section (BJD 2456928-2456988) and 0.0297647(5) $\mathrm{d}$ in the last section (BJD 2456988-2457048). We confirmed that the period had gradually decreased in the fading tail. If these modulations were not superhumps but originated by orbital motions, the periods are needed to be a constant value, thus these modulations are indeed superhumps. After the seasonal gap (BJD 2457240-2457354), 
the superhump period was estimated to $0.0297637(7) \mathrm{d}$ (the right panel of figure 4 ). This period is the almost same as the last section in the fading tail. Furthermore, the phase-averaged profiles of superhumps had also varied throughout the superoutburst (see lower panels of figure 3 and $\mathbf{A}$ ).

These results imply the possibility that these rebrightenings were mainly caused by the disk instability model. If these rebrightenings were due to the enhanced mass transfer, we should detect the signal of orbital motions.
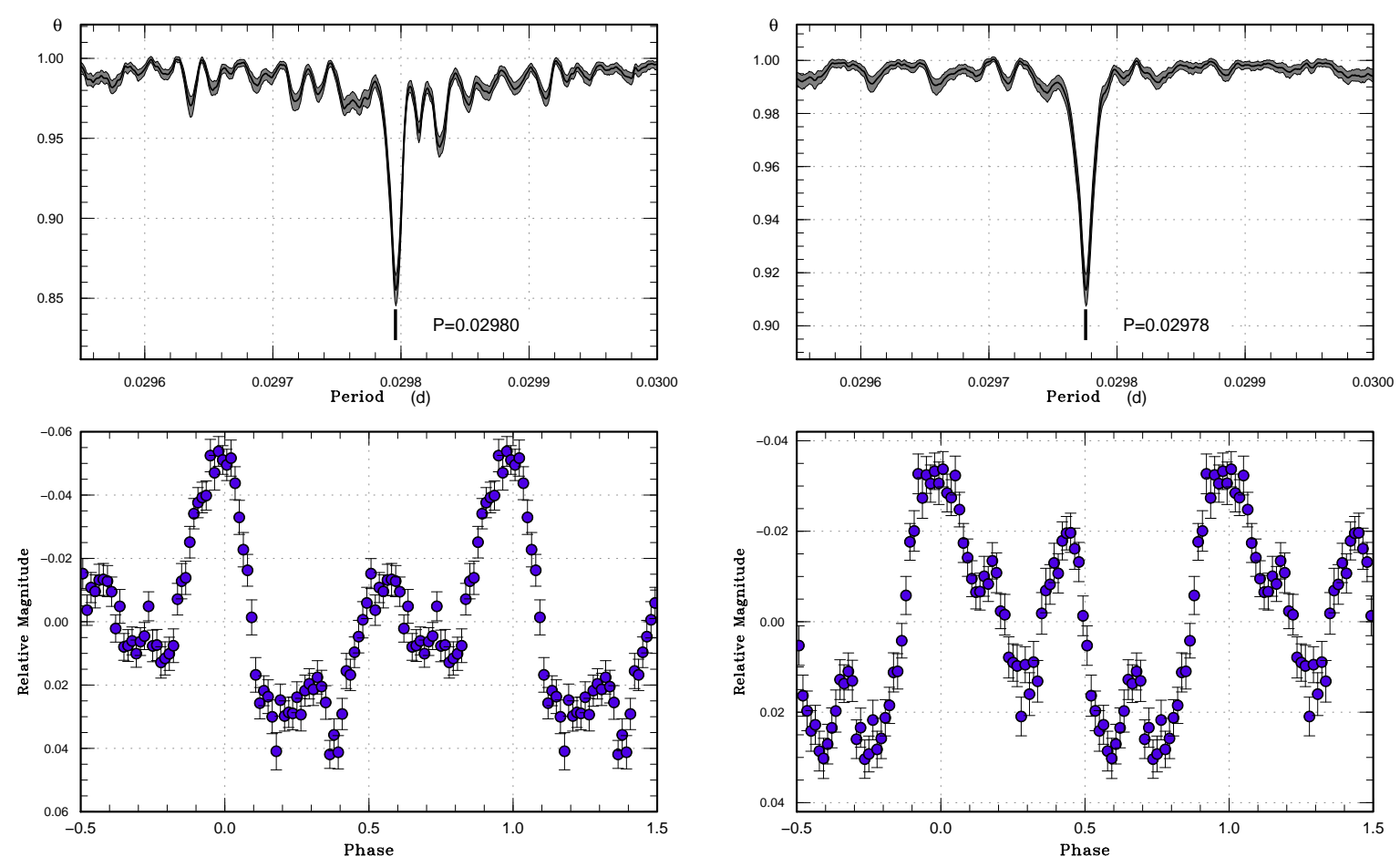

Figure 3: Results of the period analyses of superhumps of ASASSN-14ei in BJD 2456868-2456928 (Left) and in BJD 2456928-2456988 (Right). (Upper): $\theta$ diagram of our PDM analysis of superhumps. The area of gray scale means $1 \sigma$ errors. (Lower): Phase-averaged profiles of superhumps.

\subsection{Disk Radius and Late-stage Superhumps}

[[] supposed that we would estimate the disk radius by using $q, P_{\text {orb }}$ and the period of superhumps long after the superoutburst $P_{\mathrm{SH}}$, called "late-stage superhumps". Since the disk in such quiescence-like state appears to be sufficiently cold, we can ignore the pressure effect in the disk, then the fractional superhump excess $\varepsilon^{*}=P_{\mathrm{orb}} / P_{\mathrm{SH}}$ coincides with the ratio of the dynamical precession rate $\omega_{\mathrm{dyn}}$ at the disk radius $r$ and the orbital angular frequency $\omega_{\text {orb }}$ :

$$
\varepsilon^{*}=\frac{\omega_{\mathrm{dyn}}}{\omega_{\mathrm{orb}}}=\frac{q}{\sqrt{1+q}}\left(\frac{1}{4} \frac{1}{\sqrt{r}} b_{3 / 2}^{(1)}\right),
$$

where $\frac{1}{2} b_{s / 2}^{(1)}$ is the Laplace coefficient [2]],

$$
\frac{1}{2} b_{s / 2}^{(j)}(r)=\frac{1}{2 \pi} \int_{0}^{2 \pi} \frac{\cos (j \phi) d \phi}{\left(1+r^{2}-2 r \cos \phi\right)^{s / 2}} .
$$



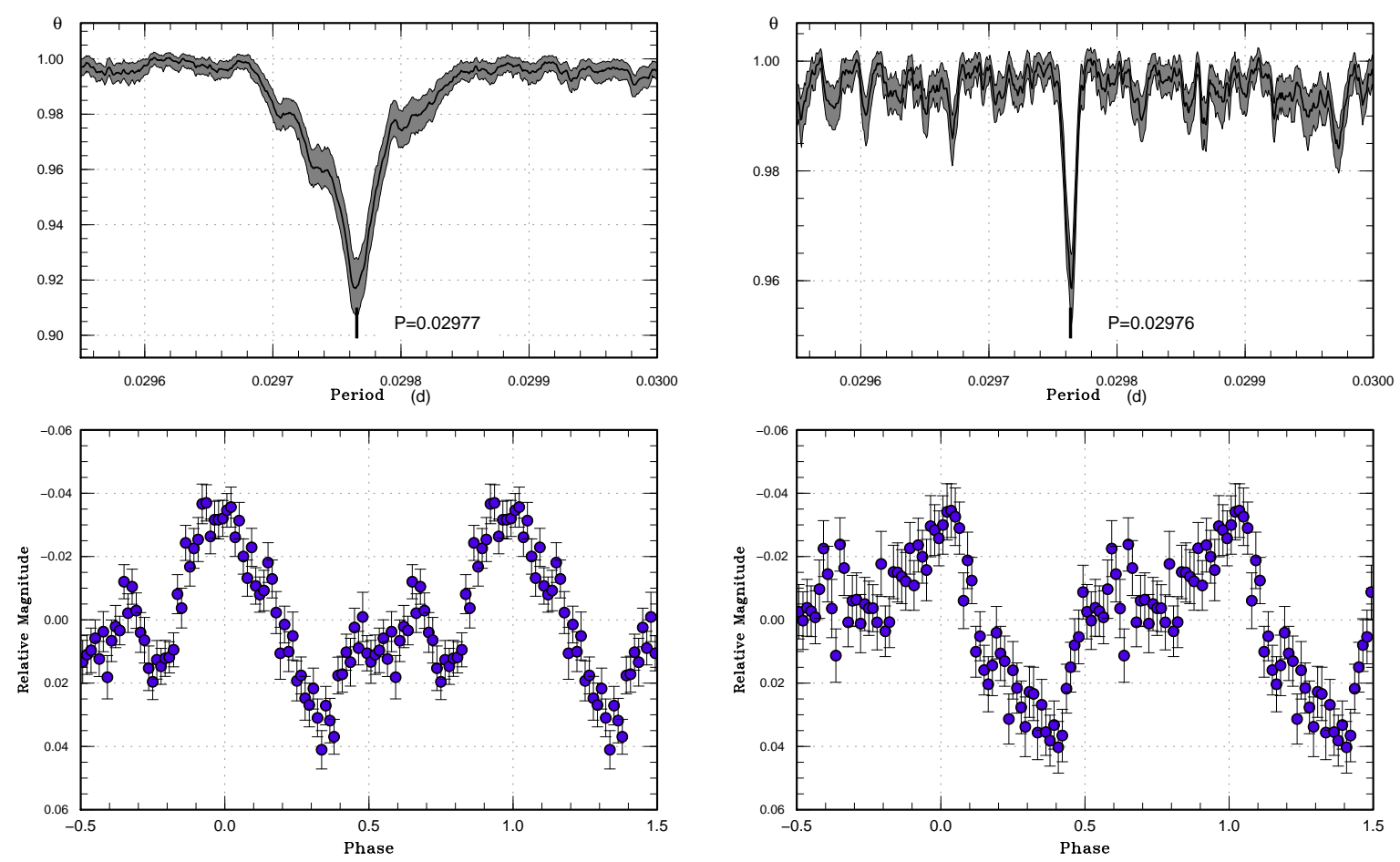

Figure 4: The same as figure 3, but in BJD 2456988-2457048 (Left) and BJD 2457240-2457354 after the seasonal gap (Right).

By using these equations, we can obtain the disk radius in the late-stage superhump phase. Unfortunately, we don't known $q$ and $P_{\text {orb }}$ of ASASSN-14ei, however the decreasing $P_{\mathrm{SH}}$ denotes the disk shrinkage. To verify the above hypothesis further observational studies of rebrightening objects are expected. The investigation of the variation of the disk radius after the superoutburst will help us to understand the mechanism of superoutburst and rebrightenings.

\section{CR Boo}

CR Boo has two distinct states: (1) fainter quiescence with regular superoutbursts state like ER UMa-type objects and (2) brighter quiescence with frequent outbursts state [22], [[2]]. In 2014, CR Boo was the former state.

\subsection{Overall Light Curve}

Figure 5 shows the overall light curve of CR Boo in 2014 April-May. The superoutburst lasted for 17 days. The inset of figure $\square$ shows the enlarged light curve during the rising part of the superoutburst (BJD 2456758.0-2456758.4), in which we can clearly see the growing superhumps.

\subsection{Stage A Superhump and Mass Ratio}

By using the PDM method and the $O-C$ diagrams, we succeeded in detecting the stage A, $\mathrm{B}$ and $\mathrm{C}$ superhumps, which is the well-known classification of the hydrogen-rich CVs super- 


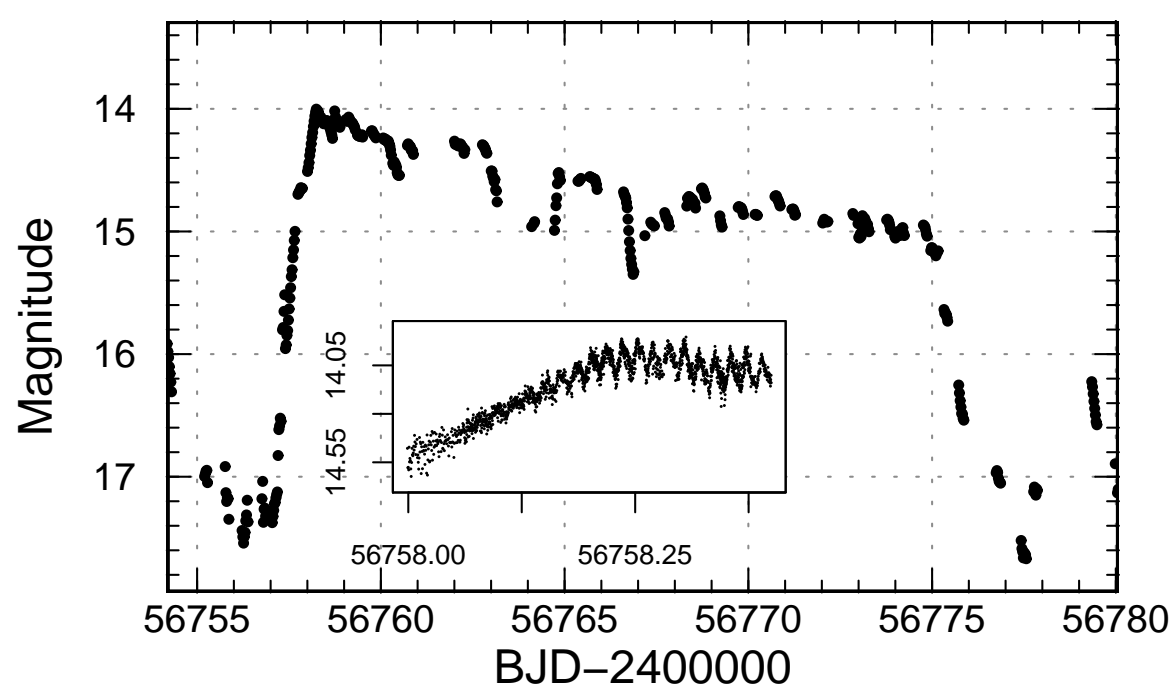

Figure 5: Overall light curve of CR Boo in 2014 April-May. Insets: the enlarged light curve corresponding to the stage A and B superhumps (BJD 2456758.0-2456758.4).

humps [24], for the second time in AM CVn-type objects. Such stage transitions suggests that the superoutburst of CR Boo is hydrogen-rich dwarf nova outbursts analogues. The estimated period of the stage A in BJD 2456758.0-2456758.195 is 0.017669(24) d. By using this period and $P_{\text {orb }}=0.0170290(6) d[$ [25], we obtained the mass ratio of CR Boo as 0.101(4).

\subsection{Evolutionary Model}

Assuming the Roche-lobe overflow and the mass-radius relation of helium-rich secondaries, [26] introduced the theoretical equations of AM CVn-type objects:

$$
\begin{gathered}
M_{2}=0.0069 P_{\text {orb }}^{-1.22} \text { for the fully }- \text { degeneratesecondary } \\
M_{2}=0.018 P_{\text {orb }}^{-1.27} \text { for the partially }- \text { degeneratesecondary, }
\end{gathered}
$$

where $M_{2}$ is the secondary mass of the system. Based on these equations, we can draw the theoretical evolutionary tracks (figure 6 ). The green dashed curves indicate semi-degenerate secondaries, and the red solid curves indicate fully-degenerate secondaries. From top to bottom, three lines represent the $q-P_{\text {orb }}$ relation, assuming $M_{1}=0.60,0.75$, and $1.00 M_{\odot}$, respectively. Figure 6 suggests the secondary of CR Boo is semi-degenerate or almost non-degenerate, and we could confirm that the estimated mass ratio lies on the track.

ACKNOWLEDGEMENTS We are grateful to VSNET collaborators, and many observations from AAVSO International Database contributed by observers worldwide. We acknowledge with thanks CRTS team for observing and providing the data. We also grateful to ASAS-SN team for the first detection and disclosing the information rapidly. 


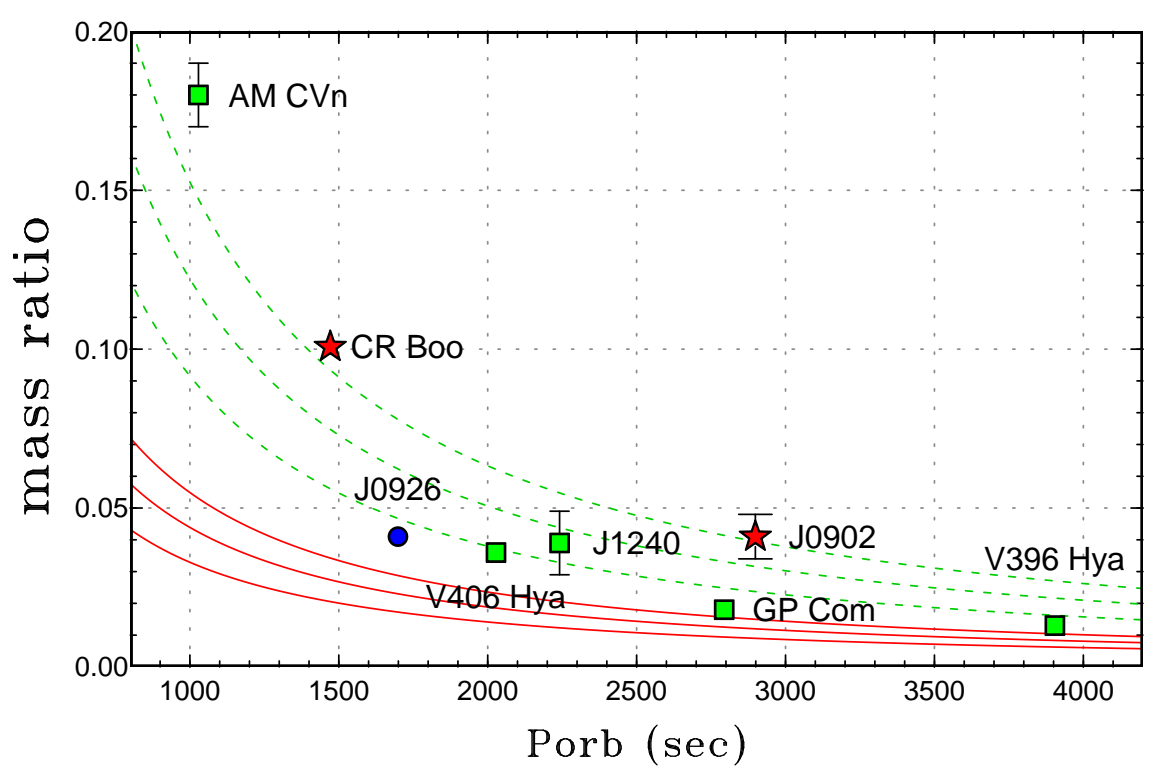

Figure 6: Relation between $q$ and $P_{\text {orb }}$ with various primary masses (same as figure 6 in [प्W]). The filled stars represent the measurements from stage A superhump (J0902 (SDSS J090221.35+381941.9): 䧃]). The filled squares represent the measurements from Doppler tomography [27] (AM CVn: [ㄹ], V406 Hya: [ㅁ]], J1240 (SDSS J124058.03015919): [B]], GP Com: [3]], V396 Hya: [四]). The filled circle represents the measurement from eclipse observations (J0926 (SDSS J092620.42+034542.3): [B2]).

\section{References}

[1] J. Solheim, PASP 122, 1133 (2010).

[2] T. Kato and Y. Osaki, PASJ 65, 115 (2013).

[3] T. Kato, A. Imada, M. Uemura, et al., PASJ 61, S395 (2009).

[4] T. Kato, T. Ohshima, D. Denisenko, et al., PASJ 66, L7 (2014).

[5] T. Kato, PASJ 67, 108 (2015).

[6] Y. Osaki, F. Meyer, and E. Meyer-Hofmeister, A\&A 370, 488 (2001).

[7] F. Meyer and E. Meyer-Hofmeister, PASJ 67, 52, 52 (2015).

[8] J.-M. Hameury, J.-P. Lasota, and B. Warner, A\&A 353, 244 (2000).

[9] B. Warner, Mem. Soc. Astron. Ital. 86, 129 (2015).

[10] K. Isogai, T. Kato, T. Ohshima, et al., PASJ 68, 64 (2016).

[11] T. Kato, M. Uemura, R. Ishioka, et al., PASJ 56, S1 (2004).

[12] B. J. Shappee, J. L. Prieto, D. Grupe, et al., ApJ 788, 48, 48 (2014).

[13] A. J. Drake, S. G. Djorgovski, A. Mahabal, et al., ApJ 696, 870 (May 2009).

[14] R. F. Stellingwerf, ApJ 224, 953 (1978).

[15] W. S. Cleveland, J. Amer. Statist. Assoc. 74, 829 (1979). 
[16] J. L. Prieto, K. Z. Stanek, C. S. Kochanek, et al., The Astronomer's Telegram 6340 (Jul. 2014).

[17] J. L. Prieto, N. Morrell, D. Grupe, et al., The Astronomer's Telegram 6475 (Sep. 2014).

[18] M. Kimura, K. Isogai, T. Kato, et al., PASJ 68, 55, 55 (2016).

[19] T. Kato, D. Nogami, K. Matsumoto, et al., PASJ 56, S109 (2004).

[20] J. Patterson, J. Kemp, D. R. Skillman, et al., PASP 110, 1290 (1998).

[21] M. Hirose and Y. Osaki, PASJ 42, 135 (1990).

[22] R. K. Honeycutt, B. R. Adams, G. W. Turner, et al., PASP 125, 126 (2013).

[23] T. Kato, F.-J. Hambsch, H. Maehara, et al., PASJ 65, 23 (2013).

[24] T. Kato, A. Imada, M. Uemura, et al., PASJ 61, S395 (2009).

[25] J. L. Provencal, D. E. Winget, R. E. Nather, et al., ApJ 480, 383 (1997).

[26] E. Armstrong, J. Patterson, and J. Kemp, MNRAS 421, 2310 (2012).

[27] T. R. Marsh and K. Horne, MNRAS 235, 269 (1988).

[28] G. H. A. Roelofs, P. J. Groot, G. Nelemans, et al., MNRAS 371, 1231 (2006).

[29] G. H. A. Roelofs, P. J. Groot, T. R. Marsh, et al., MNRAS 365, 1109 (2006).

[30] G. H. A. Roelofs, P. J. Groot, T. R. Marsh, et al., MNRAS 361, 487 (2005).

[31] T. R. Marsh, MNRAS 304, 443 (1999).

[32] C. M. Copperwheat, T. R. Marsh, S. P. Littlefair, et al., MNRAS 410, 1113 (2011).

\section{DISCUSSION}

JOSEPH PATTERSON: What data produced the CR Boo orbital period you cite? If it's photometry, it could possibly be a superhump.

KEISUKE ISOGAI: We regard the period of humps in quiescence as the orbital period, because we can always see the same period in quiescence. However, we should confirm the orbital period by spectroscopy as you say. 